\title{
Ayuda externa: ¿reduce o produce pobreza? Una aproximación conceptual a los estudios de impacto
}

René Mendoza y Klaus Kuhnekath

Los autores trabajan el tema del impacto en Centroamérica como parte de un estudio más amplio de la Universidad de Helsinki (Finlandia). Correo-electrónico: rmvidaurre@gmail.com

Recibido: noviembre de 2008 / Aceptado: julio de 2009

¿AYUDA LA AYUDA? LA RESPUESTA VARÍA SEGÚN EL TIPO DE EVALUACIÓN APLICADA: de efecto, de efectividad o de impacto. Evaluación de efecto es verificar si los objetivos y metas de un proyecto o programa fueron alcanzados al cabo de su ejecución; evaluación de efectividad es ponderar el entorno del proyecto, las políticas y el buen gobierno que influyen en su ejecución; evaluación de impacto ve los logros de metas de largo plazo, cambios en la sociedad catalizados sin que hubiesen sido planificados ni intentados por el proyecto o programa. Sobre el primero hay toneladas de estudios, sobre el segundo hay un poco menos y sobre el tercero casi no hay. Las conclusiones de las evaluaciones de efecto y efectividad, ceñidas al marco lógico, reducidas a lo técnico y económico, y limitadas por la presunción de que todos los cambios son atribuibles al proyecto, sugieren que la ayuda reduce pobreza y genera desarrollo. Si estas conclusiones fuesen ciertas, América Latina ya sería un territorio libre de pobreza.

En países con alta dependencia en recursos externos y con altos niveles de pobreza, el tema de impacto cobra realce. En este trabajo conceptualizamos impacto desde una perspectiva de economía política y de ciencias sociales, con un distintivo metodológico: que en el abanico de causas de los cambios, el contexto es previo al proyecto; es decir, ofrecemos un marco para trabajar la pregunta de si la ayuda externa reduce o produce pobreza.

Palabras clave: ayuda externa / impacto / pobreza

Einstein: Si no conocemos los orígenes, ¿̇cómo saber hacia dónde va el universo? Bohr: La realidad no existe fuera del conocimiento. Einstein: ¿Quieres decir que la luna no existe si no la ves? Bohr: Pruébame que existe sin verla. 
Los directores de proyectos no regresan a los lugares donde ejecutaron el proyecto, evitan visitar a sus ex beneficiarios... Les da miedo encontrarlos igual que antes del proyecto y hasta peor que sus vecinos no beneficiados por el proyecto.

Peter Marchetti

\section{Introducción ${ }^{1}$}

Los recursos son escasos mientras, según la CEPAL, la pobreza y la desigualdad crecen. En consecuencia, se expande constantemente el mercado de las evaluaciones de proyectos con finalidad de reducción de pobreza. Existe una gran industria en torno a las evaluaciones de efecto y efectividad; sus conclusiones son, generalmente, que los objetivos de los proyectos fueron alcanzados; sus recomendaciones son que la transferencia de recursos continúe; y si los objetivos no fueron alcanzados es porque se requería de más recursos. Sin embargo, en reuniones informales entre el personal de la cooperación internacional y sus contrapartes nacionales se susurra que prefieren no visitar lugares y familias donde ejecutaron proyectos por temor a encontrarse la misma situación ex ante del proyecto o una situación peor en comparación con familias vecinas no beneficiadas por el proyecto.

Una hipótesis solidaria con dichos proyectos y programas sería que las evaluaciones clásicas -de efecto y de efectividad- no captan otros efectos que sí contribuyen a reducir pobreza, lo que a su vez limita el diseño -o revisión- de proyectos y programas más apropiados, y que eso les pasa porque generalmente las evaluaciones miden resultados en base a la oferta (marco lógico). Por consiguiente, los efectos que se encuentran en el nivel de la sociedad, de sus economías, y en el nivel de las instituciones de los países receptores, no son captados ni aprovechados por los proyectos/programas. De ser este el caso, conocer esa dimensión se torna fundamental para re-encauzar los proyectos y los estudios, y para crear espacios de aprendizaje ponderando el tipo de cambios que directa o indirectamente provoca la ayuda -no tanto si la ayuda ayuda, sino conocer la complejidad de los cambios institucionales- y así ganar humildad (realismo) en la focalización de la ayuda.

Captar lo habitualmente escondido -que está justo debajo de la superficie- requiere de un esfuerzo teórico, amplitud en perspectiva -incluyendo lo político- y un punto de partida metodológico opuesto al seguido por las evaluaciones clásicas, que se reducen a lo técnico y a lo administrativo, a la noción de evaluación como test para proseguir con otras fases del proyecto, a procesos despolitizados, a un enfoque desde la oferta (la intervención) y a la cultura de prisa que aparentemente la agenda del desarrollo infunde.

Necesitamos trabajar el tema del impacto en el ámbito de las ciencias sociales. Para ello, después de esta introducción, la segunda sección de este artículo conceptualiza el impacto, la tercera concierne a la metodología, la cuarta y última sección puntea algunas conclusiones a modo de hipótesis de trabajo. Hacemos notar que de parte en parte se formulan interrogantes dando sentido de cohesión al conjunto del texto y a modo de reforzar la idea de que estamos frente a un tema abierto necesitado de más pensamiento. Alertamos que este trabajo no ofrece evidencias empíricas, refiere a algunos programas de desarrollo y sus actores a modo de ilustración, y referencia interrogantes desde investigaciones que hemos realizado con el objetivo de levantar más preguntas. 


\section{Conceptualización}

El análisis de impacto difiere de las evaluaciones clásicas tanto en su alcance y carácter como en sus premisas teóricas. Se ubica en las ciencias sociales en vez de las ciencias naturales -de donde parten las evaluaciones clásicas-, corresponde más a investigación que a consultoría, y se plantea métodos de intervención que toman en cuenta su propio encrustamiento (embedded) o enraizamiento (enrooted) en los contextos. Finalmente, estos tres puntos de los estudios de impacto subyacen algo en común, la premisa de la conducibilidad (driven) de la sociedad y las implicancias que de ello se derivan.

\subsection{Diferenciando impacto de efecto y de efectividad}

La evaluación de efecto busca verificar si los objetivos y las metas de los proyectos o programas definidos en su marco lógico fueron o no alcanzados al término de su período de implementación. Esos objetivos y metas son generalmente de corto plazo y definidos antes de la ejecución del proyecto, explicitados como resultados y productos tangibles a ser cotejados con indicadores medibles. La evaluación de efectividad pondera el entorno del proyecto, las políticas y el buen gobierno, que son las condiciones bajo las que un proyecto o programa se lleva a cabo. En cambio, un estudio de impacto concierne a los logros de metas de largo plazo, cambios sostenibles generados por la intervención, que incluyen no sólo los planeados y deseados sino los no intentados y los inesperados.

Koponen et al. (2004) ${ }^{2}$ plantea que el impacto sucede a través de toda la duración de la intervención. Mientras el efecto toma tiempo para materializarse y es captado al final de una intervención, el impacto puede suceder desde el primer día en que toma idea la intervención, a lo largo del proyecto y en el plazo, como consecuencia (in)directa de la intervención -cosas o aspectos no planificados ni intentados. El impacto puede ser de diferente tipo, independientemente de que la intervención se haya dirigido a la producción (o servicios) de bienes materiales, diálogos políticos o construcción de capacidades. A diferencia del efecto, el impacto de una intervención no es anticipado, son cosas y aspectos inesperados. De éste, las preguntas clave son:

- ¿Cómo afectan los proyectos y programas su ambiente más amplio?

- ¿Cómo se traduce el crecimiento económico en beneficios y pérdidas económico-sociales tangibles al interior de los países y cómo esto afecta las relaciones de poder locales?

- ¿Cómo cambia la vida cotidiana cuando alguien sale de la pobreza?

A pesar de parecer preguntas simples, se refieren al marco del impacto, que es complejo. Los proyectos y programas no son la única causa de procesos de cambio, son uno de entre varios factores que inciden en las situaciones y procesos. Tampoco los proyectos sólo implican transferencia de recursos de países desarrollados a otros menos desarrollados, o que los recursos ejecutados se lleven a cabo linealmente -realización de tal política con tales recursos. Son más bien procesos socialmente construidos y disputados, resultado de negociaciones en los cuales los actores participantes despliegan sus agendas diversas y con intereses incluso opuestos ${ }^{3}$; es decir, tanto las organizaciones interventoras como los actores beneficiarios terminan trenzados en negociaciones de forma explícita o implícita. 
Esas situaciones, procesos y sus tensiones, claves para el análisis de impacto, indican que hay contextos que a la vez son específicos a un espacio y amplios cuando responden a la institucionalidad (cadena) de la ayuda. El contexto específico lo constituyen estructuras sociales y políticas en las que toman lugar diversas intervenciones. El contexto amplio es el conjunto de las intervenciones desde el origen de las agencias de cooperación. Obsérvese aquí la principal distinción de impacto: el contexto es clave para evitar caer en los supuestos de las evaluaciones clásicas, que es el concebir a las intervenciones como si estuviesen actuando en el vacío, como si la intervención a evaluarse fuese la única causa de cambio en las familias beneficiarias y como si la intervención fuese el inicio de la historia de dichos pueblos.

El punto irresoluto es el de la atribución, el factor causal fundamental que genera cambio en la vida de las familias. Las evaluaciones clásicas suponen tenerlo claro, pues parten de las intervenciones y miran sus logros a partir de los objetivos y metas fijados por esas mismas intervenciones a través de sus marcos lógicos. Entonces concluyen que es el proyecto (intervención) el que genera desarrollo -obviamente. En el análisis de impacto no hay una receta teórica que resuelva la cuestión de la atribución, pues no hay acuerdos de cómo las últimas y próximas causas interactúan, qué relaciones sociales y estructuras pueden llevar consigo las capacidades y poderes causales, y cómo éstos se manifiestan. La situación se complica aún más cuando el propósito es centrarse en una sola intervención y encima pretender atribuir un impacto específico a una intervención particular. Igualmente difícil es atribuir causas que generan datos agregados -por ejemplo, pobreza o bienestar social y económico. En consecuencia, para comprender el impacto de una intervención, éste tiene que ser ubicado dentro del complejo contexto de la ayuda (contextualización), y expresado en un lugar y tiempo específico (contexto específico), los que son pisos propicios para iniciar el trabajo de ponderar impacto y de establecer un marco "traje a la medida" de acuerdo a cada caso. El contexto es el que nos permite captar el significado de la ayuda.

\subsection{Impacto desde las ciencias sociales}

A diferencia del efecto, con el impacto se trata de buscar y entender el contexto ${ }^{4}$, averiguar qué pasa ahí y ponderar su fuerza estructural. Con ello se busca evitar intervenciones impuestas sin conocimiento del contexto ${ }^{5}$. Las intervenciones en un contexto conocido serían intervenciones sensibles que se introducen a un contexto fluido, intervenciones que no imponen un contexto a otro contexto ${ }^{6}$. En esta sección, más que discutir lo normativo de si hay imposición o no, buscamos captar la constelación detrás del impacto y de las mismas evaluaciones clásicas, luego analizar el carácter de doble contingencia en el que se ubican las intervenciones, y finalmente el carácter estructural del contexto.

\subsection{Carácter de conducción y dominación ${ }^{7}$}

En ciencias naturales la categoría de causalidad es lineal, la lógica es: si conocemos tal fenómeno que origina tal efecto entonces cambiamos esa causalidad. Igualmente, en las ciencias sociales existe la idea de poder causar cambios, aunque a veces resulta que no se cambia nada. En el fondo todos queremos resolver los problemas societales para una vida mejor. Sin embargo, lo común tanto en efecto y efectividad como en impacto son tres 
premisas. Mencionemos dos por el momento: la primera premisa de quienes buscan resolver los problemas societales es que es posible conducir a una sociedad, algo que subyace en ambos a pesar de sus diferencias (entre efecto e impacto), o sea, la sociedad es un objeto apto para las acciones humanas. La segunda premisa es que existen medios y caminos de un impacto (o efecto) calculable sobre este objeto de acción humana -impacto a través de una conducción ${ }^{8}$.

La idea de conducir a la sociedad es una idea que viene desde finales del siglo XVIII, pasando por toda la historia del siglo XIX y XX, siendo recibida de manera diferente en cada momento, inspirando a dictadores y a creadores de sistemas de dominación totalitario, así como a movimientos de liberación del tercer mundo. La idea sigue hasta la fecha. Los políticos, ideológicamente hablando, en cada momento estimulan la idea de la posibilidad de conducción; y lo propio hacen las agencias de cooperación.

Sin embargo ésta es una idea disputada. Existe debate entre quienes argumentan que la sociedad puede ser construida versus quienes razonan que la sociedad no es construible, conducible versus no-conducible. Lo primero despierta la ilusión total que es posible conducirla, mientras lo segundo, la idea total de que no. En la literatura sobre el postheroico (Wefer, 2004), el Estado se presenta capaz de resolver la pobreza, lo que está basado mayormente en la teoría de inter-acción, mientras la teoría de sistemas niega la posibilidad de conducción, porque la política como sistema en el cual se toman decisiones es un sistema parcial separado de otros sistemas como el de ciencias, sistema moral, sistema de educación y ambiental, y por lo tanto no puede controlar los efectos de sus acciones en esos otros sistemas, salvo controlarse a sí mismo. Cada sistema ve la ley y la aplica a su modo. Un ejemplo es cuando un Estado decide proteger a los empleados de mayor edad para que no sean despedidos de las empresas, y el efecto es que las empresas evitan contratar a las personas mayores. Es decir que el sistema político trabaja con actores que reaccionan según sus interpretaciones. Por consiguiente, hay que diferenciar entre las intenciones que hay (por ejemplo, proteger a mayores, veda forestal) y las consecuencias, así como convencernos de que es una ilusión creer que el Estado puede controlar los efectos de sus decisiones.

Otra variante de la teoría de sistemas es interpretar el acto de conducir como un resultado y asumir que se puede conducir por los sistemas sin interferencia de fuera. En el tema que nos concierne diríamos que una agencia de cooperación es capaz de auto-conducirse pero no de conducir el sistema de las estructuras locales quienes, interpretando lo que quiere la agencia desde su propio punto de vista e intereses, ejecutan los proyectos en coherencia con sus intereses. En otras palabras, cada actor hará de la idea de la agencia algo diferente. Por lo tanto, poder conducir linealmente es una ilusión, pues los actores con los recursos de la agencia hacen múltiples cosas, desde robarlos, usarlos para afianzar su poder, dar dádivas, re-dirigir hacia una clientela, invertirlo, etc. El impacto, en este sentido, son estas ideas y acciones no intentadas ni planificadas por la agencia de cooperación.

De lo anterior se deduce que cada actor, dentro de su sistema, tiene su propia perspectiva. ¿Cómo se interpretan las reacciones? Dependiendo de las reglas del juego (por la política, por las agencias de cooperación). ¿Es ésta una conducción efectiva o no? El efecto es lo que 
debe ser, lo que es y los aspectos inesperados, los que no son prueba para una conducción política. En consecuencia, no tenemos una teoría de la conducción política en el sentido de analizar los efectos de conducción en un contexto causal sistemático de todas las variables. Esto, sin duda, da puerta abierta al pesimismo y sugiere que no haya intervención externa, lo que no sería correcto. Planteamos el reto de buscar las posibilidades de conducción que tomen en cuenta la reacción (interpretación) de los actores.

¿Cuál es el impacto de nuestra forma de conducir? Necesitamos distinguir entre los actos de conducir y los que proceden del sistema político según las reacciones de otros y según sus propias interpretaciones de su misma política. Por ende, la probabilidad de contabilizar efectos calculables no es alta. Sabemos que las decisiones del sistema político van a tener efectos, pero no sabemos cuáles. También podemos decir que los efectos de conducción intentados no son per se imposibles. Se trata pues de distinguir efectos entre conducción posible y no posible.

Al hablar de impacto no nos estamos metiendo en ninguno de los extremos. Buscamos un punto intermedio, pues el extremo de no-conducibilidad es considerar que el status quo es incambiable.

Las agencias de cooperación expresan este debate también, aunque en general abrazan la idea de conducción de la sociedad, más próximo al carácter lineal. Estas organizaciones buscan saber cuánto van conduciendo para que la sociedad mejore, y en ese camino suelen preferir evaluaciones de efecto que les aseguren relativamente el no poner en riesgo la continuación del flujo-transferencia de recursos. Aunque también muchas de las agencias de cooperación han aprendido que la imposición no es lo mejor, y que es necesario entender el contexto para generar mejores cambios. El desafío, sin embargo, es que esa lucha por entender no sea tanto para medir impacto (cambio) en los sujetos, sino para hacer intervención en el contexto para que la misma agencia se integre en éste. Por ejemplo, en algunos países africanos se busca evitar las matanzas, objetivo para el cual es necesario entender el contexto en el que esas matanzas se dan, pues no están originadas por gusto o por simple falta de moral, sino que revelan procesos sociales muy complejos. Entonces, a través del entendimiento se es parte también de ese contexto mayor ${ }^{9}$.

Los estudios de impacto buscan detectar los efectos de una intervención que resultan "inesperados", "no planificados" y "no-intentados" por una política, programa o proyecto. Dichos resultantes encubren los deseos de conocer algo bueno de las acciones, pero a veces lo que sale es algo diferente. Literalmente, esto podría expresar "mala planificación”, o sea que en el fondo persiste la misma lógica de conducir la sociedad, proceso en el que algo sale mal, en el que hubo un error o algún problema técnico. Es decir, a primera vista, detrás de lo "inesperado" hay planificación; a segunda vista aparecen errores de planificación. Y así sucesivamente. De lo segundo surgen otras preguntas:

- ¿Qué es conducir a una sociedad? ¿Qué posibilidades de conducción hay? ¿Cuánto influye el contexto en la conducibilidad?

- ¿Qué mecanismos desarrollan las organizaciones que para entender el contexto incluyen su misma participación y los efectos que esa participación despierta?

- ¿Cuáles son los medios y caminos de un impacto bajo el marco de la conducibilidad? 
- ¿Cuál es la génesis de esos medios y caminos?

- ¿Cuál es el punto intermedio entre conducibilidad y auto-conducibilidad?

\subsection{Contingencia, doble contingencia y teoría de las acciones}

De la conducibilidad emana la pregunta sobre la contingencia: ¿Cuáles son las causas del cambio? ${ }^{10}$ En esta pregunta -al menos vista desde el tema del impacto- subyace la idea de que si tenemos las causas entonces podemos conducir la sociedad. Es como un doctor que examina al paciente, si tiene el diagnóstico puede recetar (conducir). Causas y no impacto. La idea de conducción subyace contingencia, y la lógica es: si la sociedad no es dada por Dios, si se puede entender (es decir captar sus causas), entonces puede ser conducida. Entendimiento tiene implícita la idea de que la sociedad es hecha por hombres y mujeres, y no por Dios. Si puede ser entendida entonces puede ser cambiada, mientras algo hecho por Dios no es entendido y puede ser cambiada sólo por Dios. Es como el capitán (gobierno) y el barco (sociedad), el capitán necesita saber hacia dónde quiere ir (meta), para alcanzar esas metas necesita medios y caminos. La conducción, por lo tanto, incluye el actor (agencia) y la capacidad de conducir hacia una meta con ciertos medios, implica la presencia de un actor racional ${ }^{11}$.

La cuestión de la racionalidad nos lleva a comprender la doble contingencia. Comencemos con una analogía: si conduciendo un vehículo choco contra un árbol, éste no hace nada, pero si el choque es contra una persona, ésta reacciona incluso con la muerte. Por ende, hay que tomar en cuenta la reacción posible de los otros, pues éstos también son sujetos. Si ego quiere conducir a alter, éste va a reaccionar, o sea que hay que tomar en cuenta la situación de doble contingencia ${ }^{12}$. Entonces no sólo es necesario tener meta y medios, sino una teoría de acciones y consecuencias de las acciones. En el caso del árbol la relación causal es que el árbol no va a reaccionar, pero ¿qué teoría causal tenemos para las relaciones sociales? Esas teorías son complejas o imposibles, o sea que hay que tomar en cuenta una multitud de consecuencias no previstas, cadenas de efectos potenciales; las teorías sobre relaciones ideales y futuras casi no son posibles. Los esfuerzos de una conducción social podrían estar basados en hipótesis inseguras. En consecuencia, acciones que quieren ser intenciones de conducción deben ser entendidas sólo como intentos de conducción. Por ejemplo, la democracia que incluye participación ciudadana es un modo de conducción. A primera vista es una forma de tomar en cuenta los intereses de los ciudadanos, pero a segunda vista aparece la noción de conducción como un concepto de dominación. Por tanto, la tercera premisa, que habíamos dejado pendiente, es que los ciudadanos no sólo dan la posibilidad de conducir sino también de dominar. Ubicándonos en un marco histórico, la conducción política es gobernar y gobernar tiene dos lados: conducción y dominación ${ }^{13}$.

El concepto de conducción societal implica que tenemos que nos enfrentamos con dos modos de conducción que tienen que ver con cómo se ve la organización interviniente y lo que busca. La primera forma de conducción societal tiene que ver con un modo reflexivo del impacto sobre las relaciones sociales, esto es, que la sociedad misma, la unidad (grupo, organización o sistema parcial societal) auto-conduciéndose son tanto el sujeto como el objeto de la conducción, la consecuencia es que la dirección del efecto de la conducción es por lo tanto circular (ver dibujo superior de la ilustración 1). Por ejemplo, una agencia de 
cooperación tiene acción, y ésta tiene un impacto que llega a ella misma, o sea que la agencia se capta como sujeto y objeto de la acción. Es una agencia que busca entender el contexto y busca entenderse incluyéndose a sí misma en el contexto -el actor interviniente pertenece al contexto. Como agencia entra al contexto, pero su misma entrada genera reacciones y hay cambios. Si dicha agencia de cooperación tiene interés en hacer algo interesante, entonces tomará en cuenta esa entrada, de que es parte del contexto (efecto circular de la ilustración 1).

La segunda forma de conducción societal tiene que ver con la semántica del concepto náutico (capitán) de la conducción, lo que sugiere un modo transitivo del impacto, es decir, sujeto y objeto son entidades sociales diferentes y la dirección del efecto de la acción es lineal: un lado conduce y el otro lado reacciona (ver dibujo inferior en la ilustración 1). Aquí la agencia de cooperación pone causa, pero

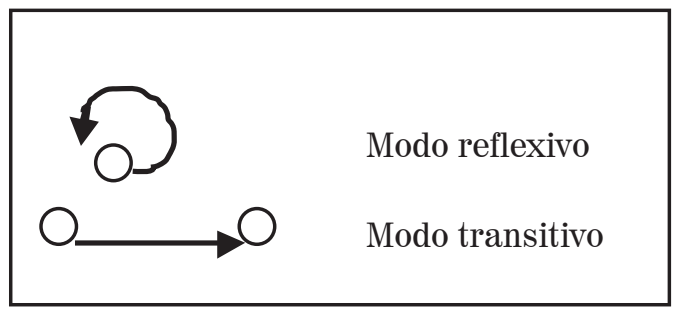

Ilustración 1. Modos de conducción entre sujeto y objeto no hay relación. La misma agencia no se considera objeto, sino sujeto, y quiere saber cuál es el impacto de su acción. Este supuesto es próximo a la analogía que hicimos con el árbol que no reacciona. De la misma manera, el otro "golpeado" reacciona pero en el modo que la agencia quiere -según sus metas y objetivos. La agencia entra sin tomar en cuenta a la gente que reacciona, los ve como si fueran un árbol -incluso usando métodos participativos.

El marco desarrollado hasta aquí nos arroja nuevas preguntas:

- ¿Es posible la conducción de la sociedad? ¿Significa el concepto de la conducción algo ideal?

- Si la conducción de una sociedad es dada, ¿cuáles son las premisas y limitaciones de esa posibilidad? ¿En qué formas y en qué intensidad puede esa posibilidad ser esperada?

- ¿Qué institucionalidad subyacente impide la primacía del modo reflexivo-circular de conducción?

\subsection{Dimensión estructural del contexto y del cambio institucional}

Iniciamos esta parte conceptual distinguiendo el impacto por la importancia que adquiere el contexto, luego repasamos los supuestos del impacto. Ahora necesitamos concentrarnos en el tema del contexto. Partamos con el viejo maestro Marx, quien de alguna forma discute también el tema del impacto, siendo su consejo que no es posible cambiar las estructuras de la noche a la mañana sin tomar en cuenta las instituciones (Marx se refería a las instituciones del capitalismo). Estamos pues frente al contexto institucional, algo tan estudiado por mucho tiempo pero que paradójicamente no ha calado tanto en nuestras mentes. ¿Por qué? Las agencias de cooperación y sus contrapartes siguen teniendo problemas en conocer ese contexto institucional y en tomarlo en cuenta, situación que en gran medida -y es nuestra hipótesis- tiene que ver con la perspectiva euro-céntrica de los organismos que intervienen, perspectiva desde la cual se ve que todo está claro, se asume que ya se conoce todo ${ }^{14}$, y las agencias emplean generalmente políticas basadas en la teoría de la modernización. 
Tampoco las ciencias sociales están exentas de esa trampa del euro-centrismo. En Europa se suele pensar en un caso europeo y fácilmente se concluye que "todo el mundo piensa así", como si Europa o Estados Unidos fuesen el centro del mundo. A esa perspectiva contribuye el hecho de que hay tanta información cuantitativa y de sistemas de información geográfica sobre niveles de pobreza, producción y población. Disponiendo de esos datos es tentador pensar que ya saben, que no hay nada que aprender de los países del sur. Esta perspectiva, contrapuesta con lo avanzado en páginas previas, nos provee razones adicionales de por qué las políticas de reducción de la pobreza suelen más bien profundizar la pobreza. Mendoza y Kuhnekath (2008) encuentran que mientras los expertos en desarrollo se equivocan, las mujeres emprendedoras son excepcionales en entender y usar ese contexto institucional ${ }^{15}$. Pero ellas representan un espacio en donde generalmente no están las agencias de cooperación, que más bien contribuyen a marginarla más. Igualmente, Mendoza (2008), estudiando impacto en Las Segovias, revela que las estructuras sociales no están incluidas en esos datos agregados, que más bien estos datos dibujan fuegos artificiales que esconden una "caja negra" (black box). Para el tema del impacto se requiere descifrar esa "caja negra".

La "caja negra" puede ser descifrada distinguiendo entre estructura aparente y estructura profunda, distinción que fue dilucidada por Levi-Strauss. Este reconocido cientista empleó la imagen de un cruce de calles donde hay una cámara para registrar los cambios sucedidos en el día y en la noche. El resultado fue que hay más gente y más carros en las mañanas, pero que a lo largo del día, el cruce de calles era el mismo. El cruce de calles sería la estructura profunda y el movimiento de gente y carros la estructura aparente. Haciendo de esto un paralelismo con Centroamérica, podemos apreciar de forma general que hay fuerte cambio en la región, cambios en las leyes, acuerdos, flujo de capital, cambios de gobiernos con diferente signo ideológico, de multitud de proyectos, pero que al final del día, las estructuras de dominación generadoras de inequidad persisten -se cambia todo para no cambiar nada.

Vinculando este punto con la idea de ver los resultados de intervenciones de desarrollo como formas de conducción, ¿es la conducción principalmente buena o mala? Dietrich Dörner (1996) escribe sobre cómo los expertos en desarrollo desarrollan fracasos: "La lógica del fracaso, pensamiento estratégico en situaciones complejas”. ¿Qué espacios de decisiones políticas tienen los otros sistemas? Recordemos que grupos sociales como los empresarios son capaces de incidir y hacer que el Estado apruebe determinadas leyes a su favor en nombre del país, de la libertad, de la justicia y de la equidad, por lo que no es el Estado el que decide sino que aprueba leyes en reacción a decisiones de grupos económicos. En este caso, el cruce de calles tiene que ver más con ese grupo empresarial y no tanto con el sistema político formal. Siguiendo con lo planteado por Levi-Strauss, la racionalidad del sistema político cambia con cada nuevo gobierno, mientras la racionalidad de mafia sigue constante ${ }^{16}$. Dicho de otra manera, los cambios políticos son cambios formales con reglas formales, mientras que las estructuras socio-económicas se refieren a estructuras inalterables, a reglas informales actuando detrás de las reglas formales o manipulándolas. Por ende, hay que averiguar qué es lo que constituye el cruce de calle como la clave del contexto. 
Finalmente, las experiencias concretas tienen lugar en un marco de estructura-agencia (structure-agency). El desafío mayor es producir puentes, de tal manera que se pueda ir de estructura-agencia hacia las experiencias concretas y de los eventos hacia ese marco. No se trata de ver la realidad sólo desde las estructuras, lo que infunde determinismo, como si los seres humanos no decidiese (agencia). Tampoco se trata de verla sólo desde la agencia, lo que infunde voluntarismo, como si las estructuras no existiesen. El hilo conductor de la investigación es que todos los actores se ven legitimados por otros. En este sentido, las agencias de cooperación se ubican en esa relación estructura-agencia, tienen agenda (agencia) y como tal precisamos conocer su génesis ${ }^{17}$ (estructuras), y cuando se presentan como "representantes de los pobres" necesitamos auscultar de dónde viene esa legitimación.

\section{Una metodología para el análisis del impacto}

Con la cuestión del contexto podemos entrar a la parte metodológica, primero a la epistemología, luego a la especificidad del método para el tema del impacto, y finalmente esbozamos la necesidad de hacer varios estudios como un método de análisis de impacto que mejore las intervenciones.

Las evaluaciones clásicas buscan atribuir los cambios sucedidos a la acción de un proyecto/ programa, ubicándose de esta manera en el marco de la epistemología del positivismo inspirada de las ciencias naturales (ver ilustración 2, $\mathrm{C}=$ contexto, $\mathrm{I}=$ intervención). En estas evaluaciones se elabora una situación con/sin intervención (situación y población similar pero con ausencia de equis intervención o política), unidades de análisis comparativo y comparación de antes/después de la intervención con la misma población. El problema es el de la atribución, ¿cómo atribuimos un efecto particular a una causa particular? Esta metodología descansa en el supuesto ceteris paribus.

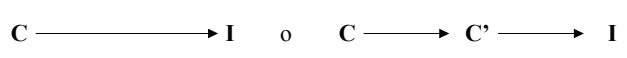

Ilustración 2. Causalidad sucesionista

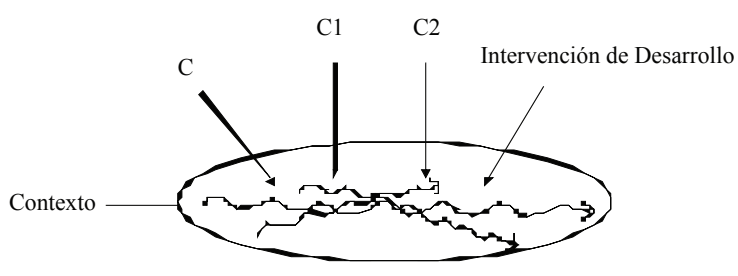

Ilustración 3. Causalidad generativa
Dichos supuestos están lejos de ser no problemáticos. ¿Qué variables pueden llevarnos a concluir que dos grupos de población son similares? Nos es conocido que las sociedades son complejas y son sistemas abiertos. La causalidad en la sociedad y en las ciencias sociales trabaja de una manera diferente de la causalidad en la naturaleza y en las ciencias naturales. En lugar de cadenas de causas seguidas invariablemente por sus efectos, el proceso de desarrollo es un flujo de un número de factores causales, factores condicionantes, determinantes y factores influyentes en el flujo de eventos. Las mismas causas pueden conducir a diferentes resultados en diferentes contextos. De ahí que atribuir una causa a un efecto es cuestionable. 
Además estamos frente a la ayuda, que es transferencia de recursos de países desarrollados a países menos desarrollados, transferencia que no se da de forma lineal sino que es un proceso socialmente negociado y disputado en el acceso a tales recursos.

En consecuencia, el método específico para el análisis de impacto es el llamado "estudios de impacto en contextos específicos" (context-specific impact studies). No se trata de partir de la intervención y seguirla para captar su impacto, sino de examinar el contexto (estructuras políticas y sociales) -identificar "el cruce de calles"- donde toma lugar la intervención, ver qué cambios -geográficos y sociales- se van dando allí y pensar cuáles mecanismos sociales están produciendo esos cambios. Eso es entender el contexto, partir de ello hacia la o las intervenciones.

La intervención no entra en un vacío, sino que el impacto se produce en una relación entre la intervención y los procesos que ya se van dando en el lugar. Es decir que la historia de los lugares y familias beneficiarias de la intervención comenzó antes de la intervención. De ahí que, a diferencia de la perspectiva sucesionista, se propone la perspectiva generativa (ver ilustración $3^{18}$ ), en la cual se percibe que las intervenciones trabajan entre varias causas y a través de un sistema de mecanismos parcialmente invisible (Pawson \& Tilley, 1997). Dado este paso, recién se trata de discernir qué contribución pudo generar la intervención equis a los cambios. Antes que la atribución va la contextualización. Ver la ilustración 4 como ilustración del impacto de una intervención, en ese caso de PROCAFOR.

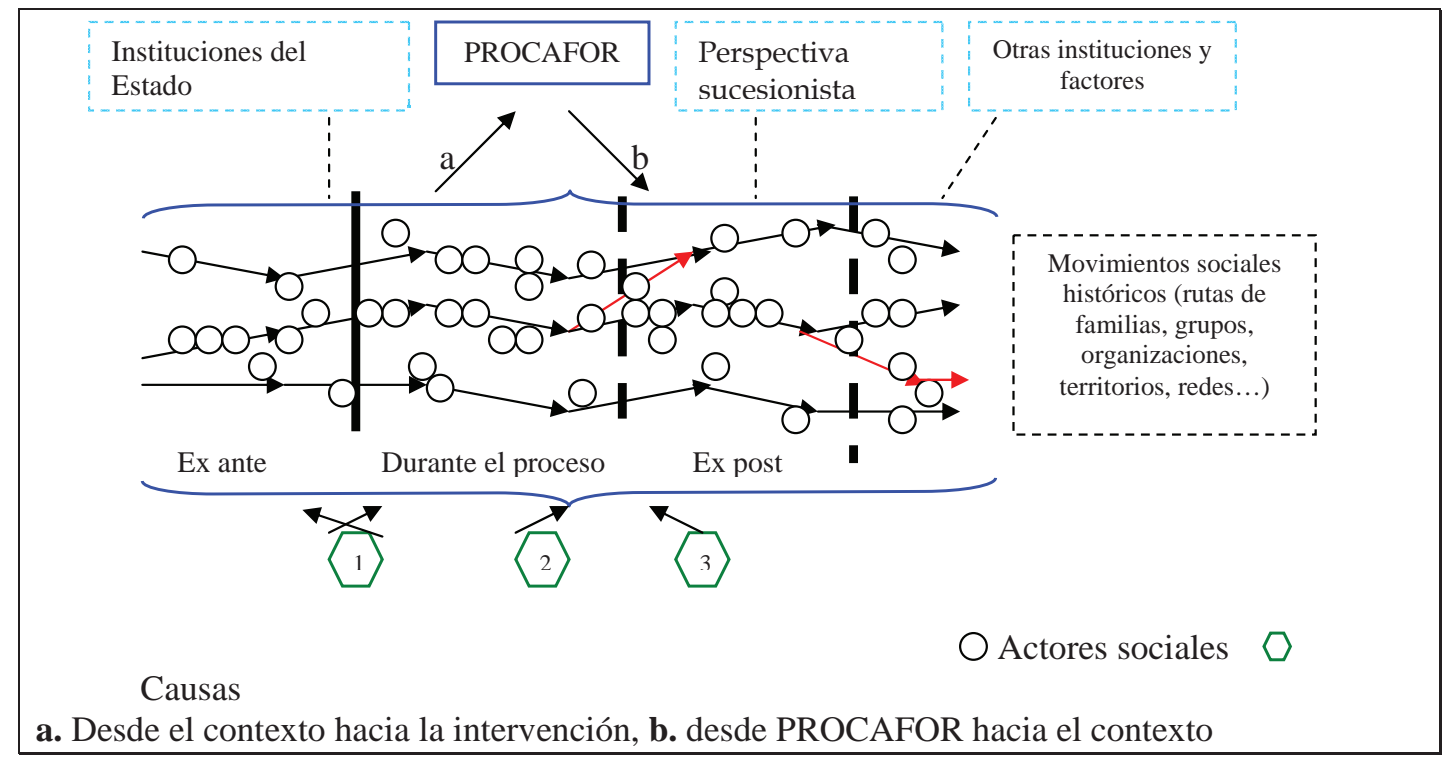

Ilustración 4. Impacto de PROCAFOR en Las Segovias

Con esta metodología, lo primero a examinar son los cambios mayores que han sucedido en el área geográfica y social donde la intervención toma lugar, cambios desde el "cruce de calles”. A diferencia de los fuegos artificiales nos referimos a cambios institucionales. Sólo 
después se intenta ver cuál pudo ser la contribución de la intervención en esos cambios. El contexto va antes que la atribución.

No necesitamos construir hechos opuestos, sino entender cómo trabajan las sociedades y qué tipo de causas operan allí. Ver causas estructurales, causas circunstanciales, causas próximas y causas de larga duración y cómo interactúan esas causas. Aquí recuperamos el aporte de Folke (2001:17-18), de intervención-contexto-proceso-impacto. La intervención es vista como un elemento dentro de muchos elementos que generan cambios. Este análisis, además, estimula el uso de cuantas técnicas cuantitativas y cualitativas puedan darse para el análisis de impacto. Como dice Folke (2001:17-18), no hay un método estándar para el análisis de impacto, éste tiene que ser construido como un "traje a la medida" en cada caso para revelar las relaciones entre intervención, contexto, proceso e impacto, con un fuerte énfasis en la especificidad del contexto.

Finalmente, es necesario no reducirnos a un análisis de impacto único, que sería como ver una estrella en medio de las nubes. Se deben hacer varios estudios de impacto para captar una constelación de estrellas a cielo abierto y así tener un patrón común que genere lecciones con directa incidencia en las intervenciones. Los estudios deben ir crecientemente combinados con sistemas de monitoreo y seguimiento llevado a cabo por los mismos proyectos y programas, de tal manera que los procesos de “conducción” sean más que todo "instituciones de aprendizaje”. Los análisis de impacto deben incluir las rendijas a través de las cuales los actores van escalando, captando los cambios institucionales.

\section{Conclusiones}

Este artículo no ofrece una respuesta a la pregunta de si la ayuda reduce o produce pobreza, pero da un marco para trabajarla. Parafraseando a Chesterton, la investigación no implica que seamos "bomberos" acudiendo a apagar cada incendio. Buscamos construir "un sistema hidráulico" que permita resolver los incendios de modo más estructural. Ante esa gran tarea, este artículo ofrece su grano de arena.

El análisis de impacto no es una ampliación de las evaluaciones de efecto. Recurre a teorías y enfoques, se replantea desde las ciencias sociales, nos devuelve interrogantes fuertes de si estamos conscientes de nuestras opciones de "conducir" y "dominar" las sociedades a través de los proyectos. Busca entender contextos como estructuras duras y ausculta cambios institucionales, marco en el cual se pondera la atribución.

Nos devuelve el tema del impacto al marco de las ciencias sociales para reflexionar dentro del "cuadro grande" y rescatarlo de las cajas técnicas y apolíticas en las que está recluido. Este artículo revela la importancia de entender lo técnico desde las novedades sociales, desde los cambios sociales y estructurales.

La importancia del tema del impacto radica en lo siguiente: si los pobres son determinados políticamente y definidos desde estructuras sociales duras, ¿cómo podrán escapar de la pobreza? Las agencias de cooperación que no se planteen estos temas y no busquen entender 
el contexto ni observarse ellos mismos como participantes del contexto, corren el riesgo de estar produciendo -y no reduciendo- pobreza.

Finalmente, este tipo de investigación debe ser necesariamente trans-disciplinaria, con primacía en uso de teorías, de diálogo con diferentes enfoques para desde ahí establecer canales de reflexión entre diferentes actores. Esto provee bases para tomar en cuenta a los actores en el proceso de los estudios de impacto.

Notas

1 Los autores quedan agradecidos por cualquier comentario y/o sugerencia que puedan recibir.

2 Juhani Koponen es Profesor en la Universidad de Helsinki y es líder de un equipo internacional para estudios de impacto de la ayuda finlandesa en varios países de África, Asia y América Latina. El texto referido es la propuesta que resultó ganadora en el concurso académico en Finlandia, correspondiente al período 2005-08.

3 Para la noción de intervención hacemos nuestro lo planteado por el prof. Norman Long: Rather than viewing intervention as the implementation of a plan for action, it should be visualised as an ongoing transformational process in which different actor interests and struggles are located. Integral to this type of approach are two other crucial aspects: an understanding of the processes by which knowledge is negotiated and jointly created throughout various types of social encounter, and an understanding of the power dynamics involved (Long \& Long, 1992:9).

4 Entender que los cambios institucionales o el status quo son resultado de las acciones humanas y no resultados naturales o producto de alguna divinidad.

5 Por ejemplo, Estados Unidos, antes de iniciar la guerra en Irak, no buscó entender el contexto de Irak. Prefirieron confiar ciegamente en sus propias armas, supusieron que la contundencia bélica provocaría la paz y la democracia. El mundo puede apreciar los efectos de esa perspectiva y tal vez imaginarse el impacto de ello en el largo plazo.

6 Ejemplos históricos tenemos en varios tipos de colonización. La española entró sin entender el contexto, imponiendo otro contexto al contexto latinoamericano. En cambio la colonización inglesa y la francesa eran más sensibles, a tal punto que buscaron apoyo en los científicos sociales para comprender el contexto. Es así como nació la antropología.

7 En esta sección nos valemos mayormente de autores como Wiesenthal (2006) y Wefer (2004)

8 Estas premisas no deben ser tomadas como algo dado. En Centroamérica existe la idea de un orden de dominación natural dado por Dios (Perez Baltodano, 2003). Bajo esa concepción las relaciones societales no son contingentes. Todo es dado por Dios, los niños son dados por Dios, los desastres naturales y hasta la política son dados por Dios. Es así que no queda lugar para los mortales, salvo aguantar. En la misma Europa, después del descubrimiento de la contingencia del orden social, de las relaciones sociales, nacieron dudas en la posibilidad de controlar los efectos del cambio; después de la revolución francesa, particularmente, nació la duda sobre si ya se tenía la nueva sociedad.

9 En un estudio reciente los autores trataron de entender a las mujeres emprendedoras. El desafío era entender el contexto en el cual ellas están haciendo emprendedurismo. Otros pueden argumentar que las mujeres emprendedoras sólo están prolongando el pasado, que no hay productividad ni inversión, y en consecuencia no hay necesidad de reivindicar políticas a favor de ellas más allá del simple asistencialismo. Otras organizaciones, en cambio, quieren conocer el contexto en que las emprendedoras emergen, saber cuál es la racionalidad de las mujeres. Esta búsqueda de comprensión es motivada por un supuesto de que en ello hay algo nuevo e interesante -supuesto que debería perseguir cualquier organización en sus intentos de entender cualquier contexto.

10 Esta perspectiva supone que las comunidades van cambiando (contingencia), que tienen historia, mientras algunos devotos del neoliberalismo dicen que las comunidades sólo cambian cuando llegan las empresas como McDonalds, Muchas evaluaciones de efecto suponen que las comunidades cambian no sólo con la intervención sino con la intervención específica que están evaluando -o sea que la historia de una comunidad empieza con la llegada de un proyecto específico, esto es lo que llamamos solapada presunción tecnocrática.

11 Por ejemplo, algunas de las agencias de cooperación preguntan por la racionalidad de los productores, de las PYMEs 0 específicamente de las mujeres emprendedoras. Al preguntar por su racionalidad quieren saber qué tipo de conducción tienen estos actores para introducirse en ese contexto.

12 Esta doble contingencia subyace a otra premisa, que por sí sola la sociedad (o alguna comunidad) no puede desarrollarse 
ni ser totalmente conducible. En la experiencia de fomento del desarrollo desde el Instituto de Investigación y Desarrollo Nitlapan de la Universidad Centroamericana se toma en cuenta esta doble contingencia y de ello emerge el siguiente axioma: "ayúdate que te ayudaré"; "te ayudamos para que puedas auto-ayudarte".

13 Es común escuchar en la población, refiriéndose a sus autoridades, "nos están conduciendo". Esta expresión tácitamente estaría incluyendo una segunda frase: "y dominándonos". Si tales autoridades son consideradas a la vez líderes y son quienes portan las llaves de las comunidades $u$ organizaciones (por ejemplo, cooperativas), estamos frente a estructuras de poder que conducen y dominan mientras las agencias de cooperación tienen similares ideas y prácticas, de conducir y dominar.

14 En 1994, como miembros de la dirección de Nitlapan-UCA sostuvimos una reunión con los representantes de un organismo bilateral. La reunión fue breve, a como sigue. Se nos propuso hacer una investigación, respondimos sugiriendo cambios en el contenido de la misma. Ellos reaccionaron: "tenemos un fondo para ese estudio, aunque de todas maneras ya conocemos los resultados que van a salir". Uno de nosotros replicó: "¿puedes poner por escrito lo que acabas de decir?" Ahí terminó la reunión.

15 Desde los datos agregados, las mujeres emprendedoras no aparecen vinculadas al tema de la innovación y si se las estudia se miden sus innovaciones en términos técnicos. El caso es que las innovaciones de las mujeres emprendedoras son innovaciones sociales o institucionales, desde las que emerge otro tipo de innovaciones técnicas.

16 También en formas de organización corporatista o gremial, como las cooperativas, se puede distinguir entre el "cruce de calles" y el movimiento de capital, de socios y de directivos. En ellas, es nuestra hipótesis, la estructura de poder anterior al cooperativismo no ha cambiado.

17 En nuestros días las agencias de cooperación en el mundo han abrazado la idea de alineamiento y armonización en torno a políticas sectoriales (SWAP). En ese propósito ellos van exigiendo, dando ideas, personal y recursos financieros en la elaboración de los SWAP en educación, salud y recientemente también en el sector agropecuario. Sin embargo, así como ellos exigen conocer las bases institucionales de las políticas sectoriales, las reglas del juego en el gobierno, ellos se resisten a proveer información con relación a las bases de sus políticas en sus gobiernos con relación a su apoyo de los SWAP (Castejón et al, 2007). Ellos piden transparencia a los gobiernos, pero mantienen de forma compartimentada sus políticas como agencias de cooperación. A esto nos referimos con génesis de sus agendas, sin lo cual difícilmente se pueden construir puentes entre las instituciones.

18 En un territorio social se da un contexto (C) donde se da una intervención produciendo otro contexto (C1), y luego otro (C2), tal como se aprecia en la ilustración 3. Y es en medio de esa realidad que se da la intervención que se quiere estudiar.

\section{Referencias bibliográficas}

CASTEJÓN, M.;FERNÁNDEZ, A.; MENDOZA, R.\&WIGGINS, S. (2007)Estudio del Programa Sectorial de Desarrollo Rural Productivo (PRORURAL), Managua: Plataforma Global para el Desarrollo Rural.

DÖRNER, D. (1996) The Logic of failure: Recognizing and Avoiding Error in Complex Situations. New York: Perseus Books. Originalmente publicado en Alemania en 1989 bajo el título: Die Logik des Scheiterns. Strategisches Denken in komplexen Situationen

FOLKE, S. (2001) Aid Impact: Development Interventions and Social Processes (AID). Research Programme 2001-2004. Centre for Development Reserach, Coipenhagen.

KOPONEN, J., ET AL. (2004) Does Finnish aid matter? A study on its impacts on states, forests and rural people. Finlandia: University of Helsinki, Institute of Development Studies.

LONG, N. \& LONG, A. (eds) (1992) Battlefields of Knowledge. London and New York: Routledge.

MENDOZA, R. (2008) Los guardianes del fuego en Las Segovias: ¿Cómo las realidades locales son parteras de la globalización? Mimeo.

MENDOZA, R. \& KUHNEKATH, K. (2008) Mujeres emprendedoras en Centroamérica: Espacios transnacionales en territorios nacionales. Mimeo. 
PAWSON, R. \& TILLEY, N. (1997) Realistic Evaluation. London: Sage.

PÉREZ-BALTODANO, A. (2003) Entre el Estado Conquistador y el Estado Nación: Providencialismo, pensamiento político y estructuras de poder en el desarrollo histórico de Nicaragua. Managua: IHNCA-UCA.

REINHARDT, T. (2008) Claude Lévi-Strauss zur Einführung. Hamburg.

WEFER, M. (2004) Kontingenz und Dissenz. Postheroische Perspektiven des politischen Systems. Studien zur politischen Gesellschaft, Bd. 5. WIESENTHAL, H. (2006) Gesellschaftssteuerung und gesellschaftliche Selbststeuerung. Eine Einfuehrung. 